\title{
THICK-FILM LASER TRIMMING PRINCIPLES, TECHNIQUES AND RECOMMENDATIONS
}

\author{
RIC CONRADT and LYNNE S. MARCUS
}

Teradyne, Inc., Boston, Massachusetts 02111

\begin{abstract}
Trimming plays an essential role in the manufacture of thick-film resistor networks. The process used to define circuit elements on a substrate, based on silk-screening techniques, is fast and inexpensive and has greatly increased the volume of manufactured devices. However, the same factors that contribute to making the screening process economical in terms of time and simplicity also present difficulties in creating consistently accurate devices. Because of inaccuracies and variations in the screening process, manufacturers cannot guarantee resistors to better than \pm 10 to $20 \%$ of the desired value. Trimming each resistor enables them to overcome the inherent limitations of the screening process and increase their yields of accurate and reliable devices. The laser has become an effective tool for trimming resistors because it is fast as well as accurate and can be controlled in order to produce consistent results. The following article discusses the objectives and principles behind laser trimming, the variables that affect the ability to produce an accurate trim, and different trimming techniques. It also offers recommendations for achieving optimum trimming speed and precision.
\end{abstract}

\section{WHY TRIM RESISTORS?}

Trimming is an essential part of manufacturing thickfilm resistor networks. The process used to define circuit elements on a substrate is based on silk-screening techniques and is fast and inexpensive. It has greatly increased the volume of manufactured devices. However, the same factors that contribute to making the screening process economical in terms of time and simplicity also present difficulties in creating consistently accurate devices.

Because of inaccuracies and variations in the screening process, manufacturers cannot guarantee resistors to better than $\pm 10-20 \%$ of the desired value. Trimming each resistor enables them to overcome the inherent limitations of the screening process and increase their yields of accurate and reliable devices. The laser has become an effective tool for trimming resistors because it is fast as well as accurate and can be controlled in order to produce consistent results.

\subsection{The Basic Objectives}

The basic objectives in resistor trimming are to provide a resistor at the desired value with the highest throughput that can be achieved and with the least effect in temperature coefficient, power dissipation, voltage coefficient, noise, and drift characteristics. The ability to trim an accurate resistor is a function of many variables. These factors generally relate to the physical characteristics of the part such as resistor size, resistor material, passivation, resistor-aspect ratio, thermal properties, and value-change required. However, other important considerations are the speed, resolution, and versatility of the measurement and beam positioning systems.

The final cutoff value set for trimming is rarely the final value desired for the part; instead it is a cutoff that enables the part to coast into its final value after the laser is turned off. For high-accuracy trims, this value is generally determined experimentally. The stability or repeatability of the measurement, however, is very important. If the cutoff occurs at the same value for every trim, it is possible to achieve high trim accuracy that is independent of the absolute value of the cutoff limit. Absolute measurement accuracy is only required to verify the part at final test after the trim is completed.

\subsection{Trim Stability}

Laser-trimmed resistors in general are less stable than untrimmed resistors. This is due to stresses and compositional changes in the resistor material brought about by the high temperatures and thermal gradients induced by the laser beam. One form of laser damage prevalent in thick film resistors is micro-cracking. Other forms of damage are not as conceptually simple but in general tend to affect the resistor at the end of the kerf or trim as the unvaporized or unmelted portion of the resistor material cools. These effects tend to cause instability and noise. Different trimming tech- 
niques, described in detail below, can compensate for these effects. For example, because the end of a straight cut is at the point of maximum current density, instability and noise increase as the trim termination approaches the opposite side of the resistor.

L-cuts usually provide better stability than straight cuts because the trim terminates parallel to the current path, and micro-cracks and stresses generated at the trim termination have less of an effect on the resistor. Laser damage is also less with double cuts because of the shorter trim length. A way to improve laser stability when using double cuts is to wait for the short-term in stability from thermal stresses to settle out before making the second cut. Performing the first cut on all resistors and then going back and making the second cuts will accomplish this. When long, narrow resistors are trimmed, however, the L-cut is better for large changes in resistor value.

\subsection{How to Increase Yields}

Many factors in the design and process steps of substrate manufacture can provide higher quality, increase yield, and greater throughput during resistor trimming. The resistor design should be as large as possible for the size of the substrate. Probe pads should be relatively flat and 20 mils square or greater in size. Resistors should be consistently fired between job lots to greater than 50 per cent of the nominal value, and they should be screened as thin as possible to maintain consistency between job lots and allow higher trim speeds. Each resistor and conductor screen operation should have the same reference edge, and resistors should not be overglazed except to meet performance parameters.

Several guidelines in trimming procedures are also useful. Trimming should affect only the amout of material necessary to obtain the desired value, and different trim techniques should be tried to reduce ruptures or cracks in the material. Power dissipation, noise, and drift must be considered if the trim length is greater than 50 per cent of the width of the resistor. The distance between trims and the edge of the resistorconductor termination should be at least 10 to 20 mils. The movement of the laser should be regulated to provide the fastest and cleanest cut, and trimming should use the same substrate reference edges that were used for screening the resistors and conductors. Finally, the laser beam focus should be optimized for the particular substrate thickness.

Trimming some substrate lots will be more difficult than trimming others because of low screened values. Different trim techniques can compensate for lower fired values or shifts in screens, but at the same time each technique may have a different effect on other parameters such as power dissipation, temperature coefficient, voltage coefficient, noise, or drift. Thus, the most efficient trimming technique will depend on several variables, and different techniques will produce different results.

\section{PRINCIPLES OF LASER TRIMMING}

Laser trimming depends on several different technologies besides those required to initially fabricate the circuit. Trimming is primarily concerned with layout, geometry, the mechanism and physics for material removal, and the measurement means for terminating the trim process. Resistor layout and geometry are determined before the wafer is fabricated. The laser and its operation establish the mechanisms and physics of material removal as well as trim termination procedures.

\subsection{Controlling the Laser Beam}

A laser utilizes a light beam to remove material from the substrate. It does this with a short-duration $(<1 \mu \mathrm{sec}$.), high-intensity, coherent light pulse that impinges on material, absorbing the light energy and causing the material to rapidly heat and vaporize. The amount of material removed by one laser pulse is typically 1 to 2 mils in diameter, although this figure will vary widely depending on the power level and focus of the laser pulse as well as the composition of the material.

There are several ways to control the laser beam. A galvanometer beam positioner, such as those used in Teradyne laser trim systems, provides a reliable means of control. Galvanometers use mirrors that can be rotated at very high speeds and at accelerations of hundreds of g's, with both long- and short-term repeatability. The galvanometer beam positioning system in Teradyne laser trim systems is digitally programmed. The galvanometer positioning control voltage originates from a $\mathrm{D} / \mathrm{A}$ converter whose resolution combined with the beam positioner field size determines the maximum positioning resolution. For example, the static resolution of a 12 -bit D/A with a $2^{\prime \prime} \times 2^{\prime \prime}$ field size is a minimum of a 0.5 mil positioning increment. The dynamic resolution can be much better than this.

The galvanometer and its mirror form a system with both mechanical and electrical inertia that combines the increments of motion to form a smooth continuous response. Simply pulsing the laser more than once for each galvanometer increment will create dynamic bite 
sizes much smaller than the static resolution. Twenty laser pulses for each galvanometer increment on the 12-bit D/A and 2 " $\times 2$ " field will produce a dynamic bite size of 0.025 mils. However, using 16-bit D/As results in a static resolution of 0.03 mils over a $2^{\prime \prime} \times 2^{\prime \prime}$ field, making more than one laser pulse per galvanometer step unnecessary. A small static bite size is important for functional trimming, which by necessity has a long measurement time, resulting in a low laser pulse rate that leaves the galvanometers unable to integrate the step function sent to them.

The effective utilization of a galvanometer beam positioning system for many applications, including active and passive trimming to ac or time-oriented measurements, is determined by several factors, especially the relationships between measurement speed of the software, the incremental movement of the galvanometers, and the frequency of the laser pulses. Experimentation is the best way to derive the most effective combination for any application.

\subsection{Determining Q-Rate and Bite-Size}

The pulse rate of the laser, or the Q-rate, and the distance the laser beam moves between each pulse, or the bite size, determine the movement speed of the laser beam. The movement speed equals the Q-rate times the bite size. In order to make a clean cut, called a kerf, the pulses must overlap. To maintain a clean kerf, the bite size should usually be less than the kerf width.

Clearly, if the laser spot size, or hole size, decreases, the kerf width also decreases, and the bite size will have to be decreased accordingly. (See Figure 1.)

In trimming high accuracy parts, very small bite sizes are necessary for reasons other than good kerf characteristics. Because measurements occur prior to each laser pulse, the amount of change in value due to the amount of material removed by one bite must be less than the desired accuracy tolerance. Otherwise, one

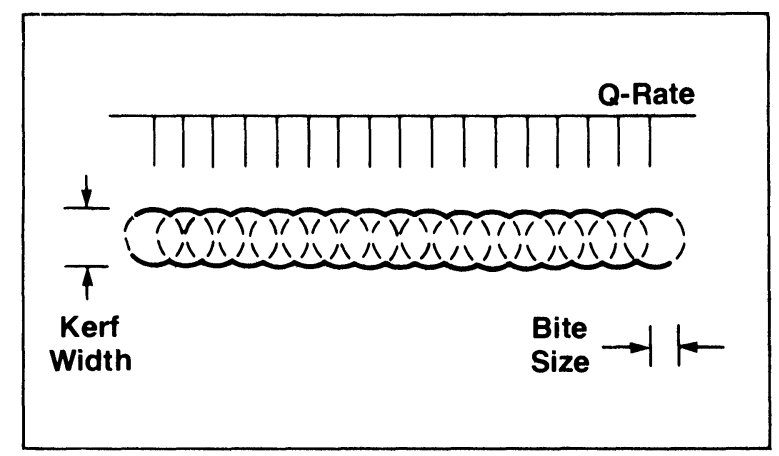

FIGURE 1 Definition of terms used in laser trimming. laser pulse may yield a value below tolerance while the next pulse removes enough material to place the value well above its tolerance. Cuts in shaded areas, areas of lower rates of change, made in addition to the main cut allow better incremental control. Furthermore, making small bite sizes and terminating the trimming procedure before the resistor reaches the desired value achieve consistently accurate trims.

\section{TYPES OF CUTS}

Most laser trims made on resistors consist of three types of cuts and their variations: straight cuts, L-cuts, and double cuts. The last category also includes doublereverse and serpentine cuts. Scan cuts are a variation of the straight cut. The geometry of the resistor and the resistivity of the film determine the resistance value of the resistor. Thus, identical geometries will achieve the same value if the film is consistent. The appropriate trim, then, depends on a combination of resistor geometry and the desired precision and speed. As with setting Q-rate, bite size, and measurement speed, experimentation can help determine the most efficient trim.

Straight cuts. The straight cut is the fastest cut in resistor trimming. It gives less precision than multiple trims, but it trims in the shortest time period. However, it is not useful for trimming a high-accuracy resistor because of the rapid change in the value of resistance (to infinity) as the cut approaches the opposite side of

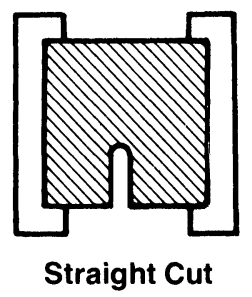

Usage: Resistor geometry of up to 2 square.

Recommendations:

- Keep trim within the center third of the resistor body to maximize value change for a given depth of cut and to allow for shifts in resistor and conductor screens.

FIGURE 2 Type of trim cut - Straight Cut. 
the resistor. If the initial value of the resistor is close to final value, the cut will stop before the point of rapid change, beyond which reasonable control and accuracy are difficult to achieve. (See Figure 2.).

L-Cuts. L-cuts overcome the problem of rapid valuechange inherent in the straight cut. They give moderate precision and a longer trim kerf by trimming straight in until the value begins to change rapidly. At this point, the trim changes direction to run along the length of the resistor for more vernier control. An added benefit of the L-cut is that it allows a greater adjustment range than the straight cut for resistors with greater than 1 -square aspect ratio (the resistor is longer than it is wide). (See Figure 3.)

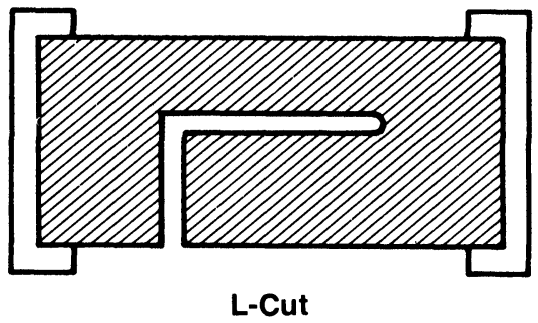

Usage: Resistor geometry of $1 \frac{1}{2}$ to 5 square.

Recommendations:

- Start trim approximately 15 to 20 per cent of the total resistor length away from the conductor-resistor junction to avoid junction effects and provide the maximum room for the second cut.

FIGURE 3 Type of trim cut - L Cut.

L-Cut with Vernier cut. This cut is used to trim resistors with geometries greater than 1 square to the nominal value desired, with more precision than a single L-cut and with generally better stability than the double cut. (See Figure 4.)

Double cuts. Double cuts give more precision than a straight cut but take more time. They also overcome the rapid-value-change problem. The first cut of the double cut brings the resistor to a value that permits the

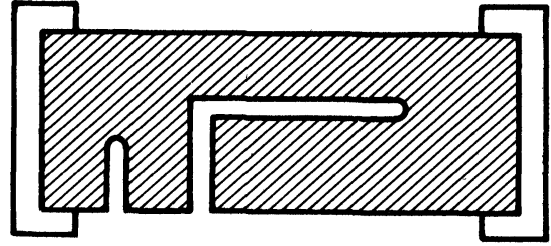

L-Cut with Vernier Cut

Usage: Resistor geometry of $1 \frac{1 / 2}{2}$ to 5 square.

Recommendations:

- Start trim approximately 15 to 20 per cent of the total resistor length away from the conductor-resistor junction to avoid junction effects and provide the maximum room for the second cut.

- The vernier trim should fall near but outside of the L-cut shadow to provide a slow rate of resistor value change. The vernier trim should bring the resistor to the last 1 to 2 per cent of the nominal value desired. The trim should not exceed the L-cut profile.

FIGURE 4 Type of trim cut $-\mathrm{L}$ Cut with Vernier Cut.

second cut to work in a lower rate-of-change area. The second cut is an additional vernier action in that it works in the shadow of the first cut. Current flows down the length of the resistor and since the second cut is made in a location where little current flow occurs, it has less effect on changing the resistor value. For this reason, the second cut should be shorter than the first cut; otherwise, vernier action is lost. (See Figure 5.)

Double-Reverse cut. The double-reverse cut is used when the screened value is lower than normal. It has the least amount of resistive material disturbance and greatly increases the value of the resistor by forcing an increase in the current-path length. This increase is a function of cut depths and locations and the doublereverse cut should avoid cut lengths that make the resistance rate of change too high. (See Figure 6.)

Many variables determine the best cut and its characteristics: resistor-aspect ratio, amount of resistor change required, resist material, final tolerance, stability, and trim throughput rate. Generally, optimizing the cut selection and its characteristics requires some experimentation. 


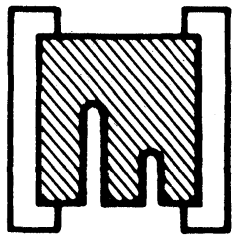

Double Cut

Usage: Resistor geometry of $1 / 4$ to 2 square.

Recommendations:

- Keep trim within the center third of the resistor body to maximize value change for a given depth of cut and to allow for shifts in resistor and conductor screens.

- Trim speed can be greater than for a single cut since precision derives from the second cut, which occurs in a less sensitive trim area than the first.

- The second trim should always be less than the first. Try to keep within 50 to 75 per cent of the first trim length for the best vernier results. At equal trim lengths, the rate of change increases rapidly and may cause overshoot and out-of-tolerance value.

FIGURE 5 Type of trim cut - Double Cut.

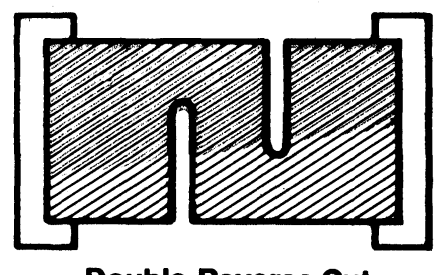

Double-Reverse Cut

Usage: Resistor geometry of 1 to 3 square.

Recommendations:

- Keep trims within the center third of the resistor body to maximize value change for a given depth of cut and to allow for shifts in resistor and conductor screens.

- Always keep trims at least 15 to 20 mils apart.

FIGURE 6 Type of trim cut - Double-Reverse Cut.

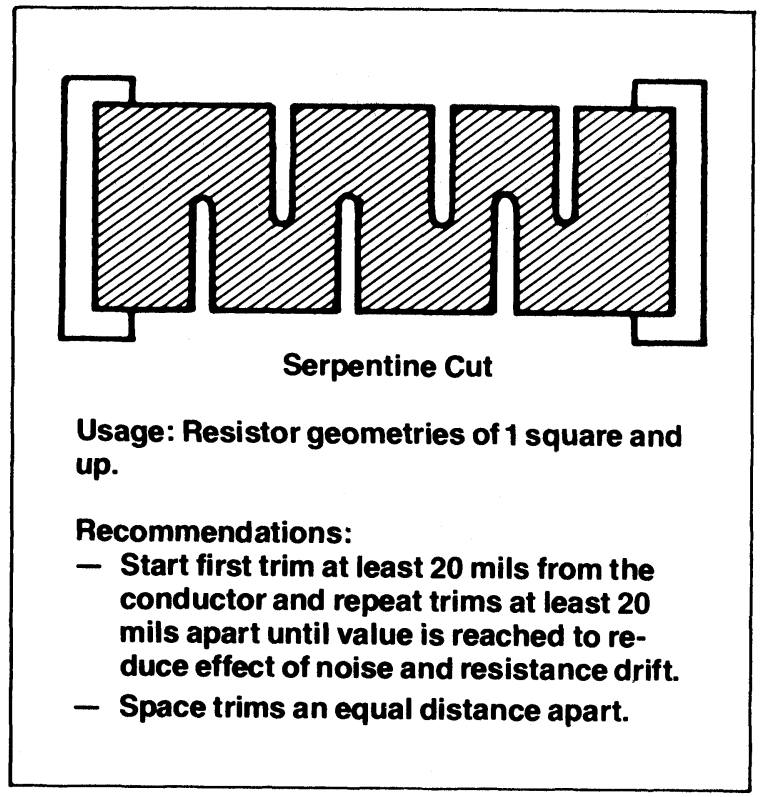

FIGURE 7 Type of trim cut - Serpentine Cut.

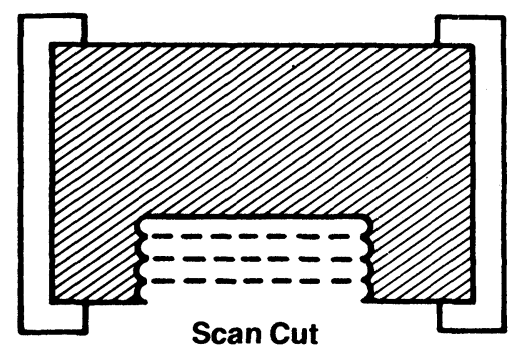

Usage: Resistor geometries of $1 / 4$ square and up.

Recommendations:

- Start three or four scans before the resistor edge.

- Use the proper step increment for a given kerf width so that all of the resistor material is removed between scan lines. For example, if the kerf is $\mathbf{2}$ mils, use a step increment of less than 2 mils to assure material removal.

- For ultimate trim stability terminate each scan, including the last, in the conductor pads. The film and conductor systems must be compatible with this trim method.

FIGURE 8 Type of trim cut - Scan Cut. 
Serpentine cut. This cut uses a maximum of six cuts to trim resistors of larger areas to a nominal resistor value that is many times that of the fired value. Serpentine cuts are not effective on small resistors because of the large area of material affected by the laser beam. (See Figure 7.)
Scan cut. A scan cut is used to sense the edge of the resistor and to trim resistors to the nominal value desired when the resistance correction is small and precision is needed. (See Figure 8.) 

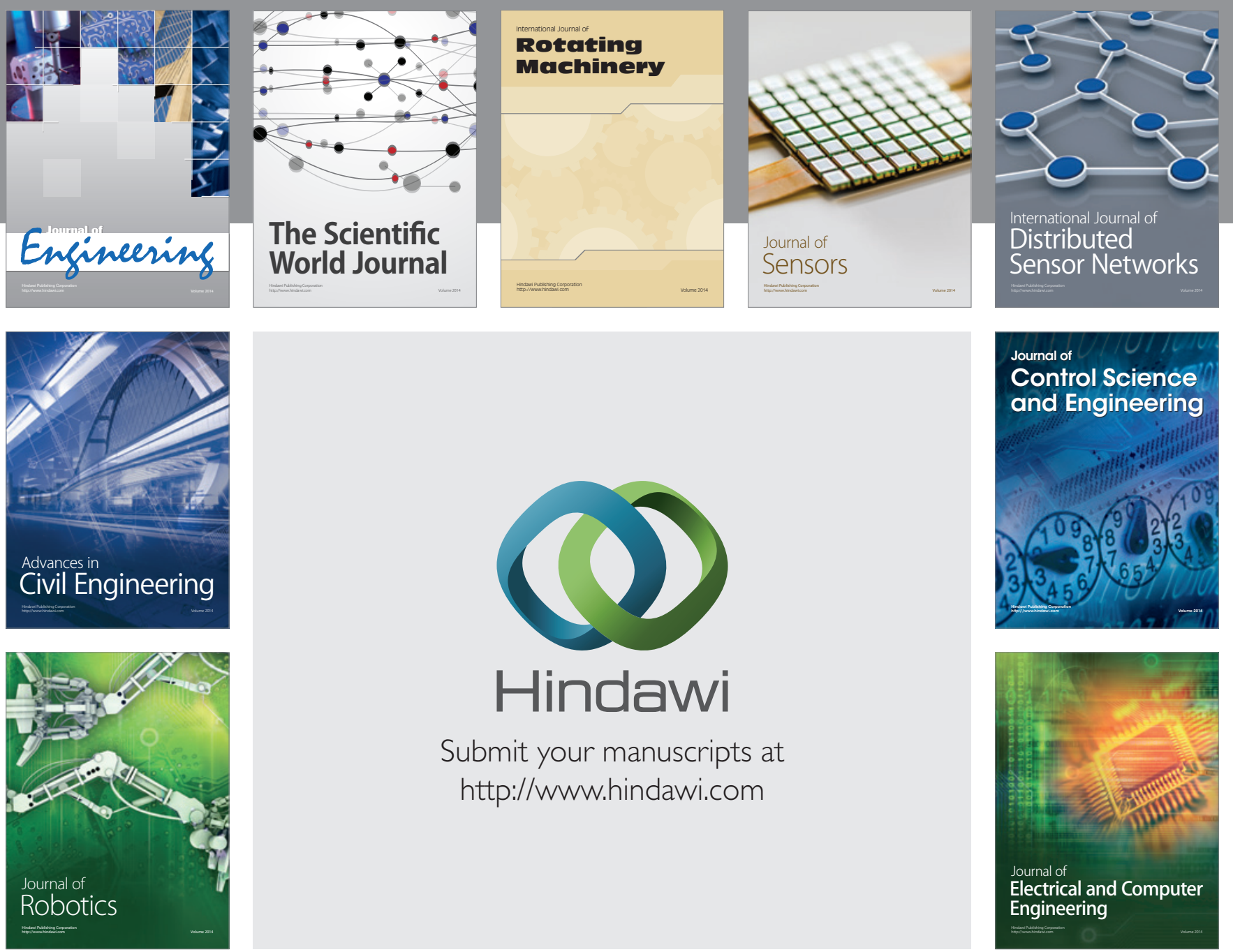

Submit your manuscripts at

http://www.hindawi.com
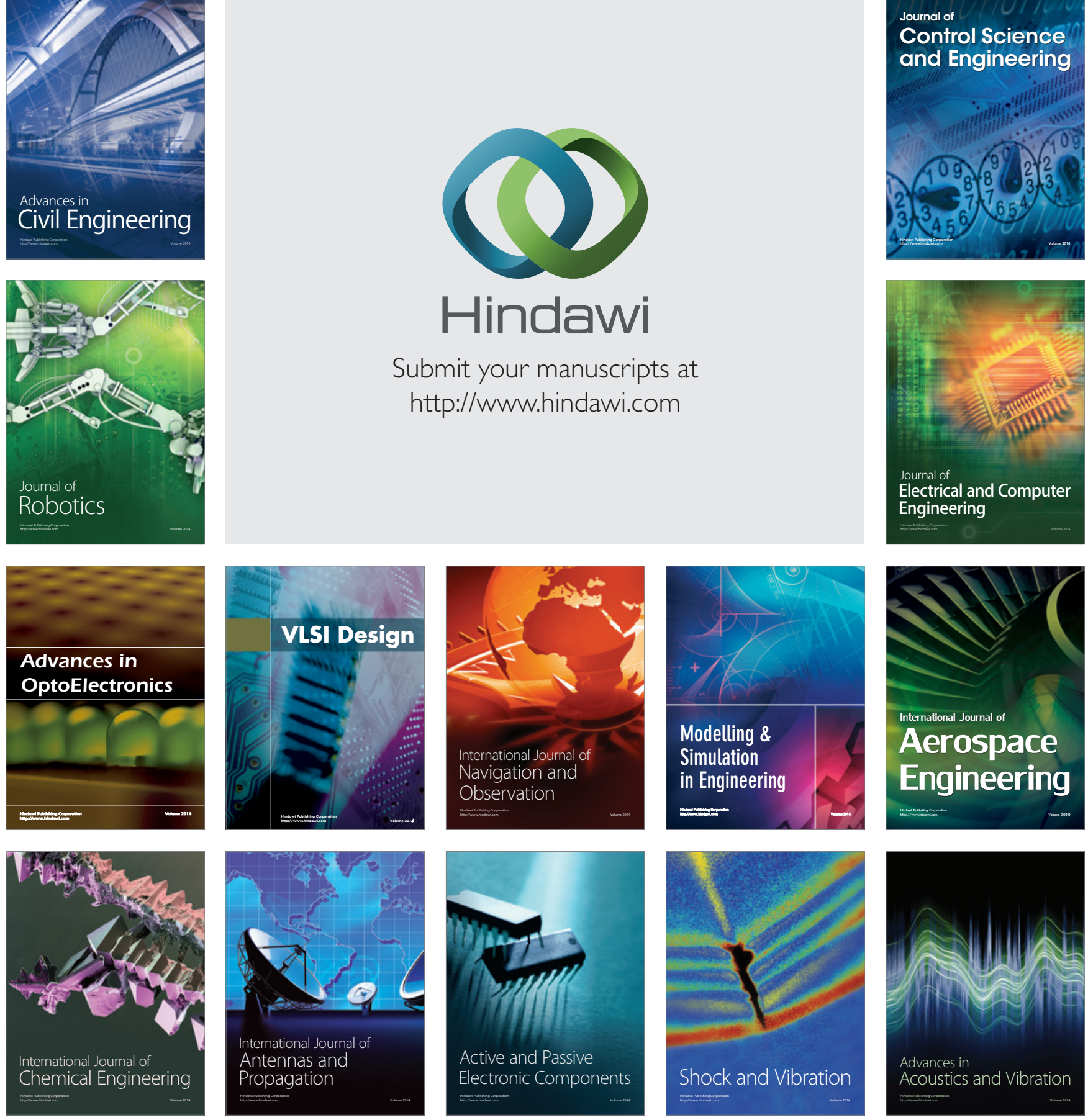